\title{
LIDERANÇA: SEU PAPEL VISANDO O CLIMA E A CULTURA NAS ORGANIZAÇÕES
}

\section{LEADERSHIP: ITS ROLE AIMED AT CLIMATE AND CULTURE IN ORGANIZATIONS}

\author{
Jonathan Laranjeira Saraiva da Silva \\ Acadêmico do $7^{0}$ período em Administração pela Alfa Unipac, Aimorés/MG, Brasil \\ E-mail: jonathan.saraiva@gmail.com
}

\section{Charleston Sperandio de Souza}

Mestre e Professor de Administração pela Alfa Unipac, Aimorés/MG, Brasil

E-mail: charleston.sperandio@yahoo.com.br

\section{Resumo}

O presente trabalho teve como objetivo descrever como a liderança, as características e habilidades do líder, a relação entre líder e seus liderados e liderança estratégica influenciam no clima e na cultura organizacional. A base teórica da pesquisa utilizou-se dos discursos sobre Liderança com foco no líder como sendo um importante elemento para que as organizações obtenham um ambiente harmonioso e saudável por meio do clima e cultura organizacional e para que seus colaboradores possam trazer melhores resultados para a empresa. A metodologia teve caráter exploratório com ponto de vista qualitativo, onde foram utilizados artigos, livros e monografias, onde está sendo relacionado no levantamento em conceitos sobre quais tipos de características e habilidades que os líderes podem exercer para que o clima e a cultura de uma organização esteja em perfeitas condições e que seus colaboradores possam desempenhar suas funções e que 0 relacionamentos entre líder e liderados estejam sempre a favor dos objetivos da organização. Com isso foi encontrado por meio das análises, que por existir uma procura por líderes pelas organizações, elas necessitam de profissionais habilitados para melhorar cada vez mais os seus resultados, conduzindo pessoas de forma positiva, influenciando-os com motivação e assim alcançarem os objetivos propostos. Conclui-se que, no ambiente empresarial é necessário que o líder obtenha a competência de influenciar de maneira positiva seus membros, onde o clima e cultura organizacional são fatores primordiais para a organização, pois os mesmos serão estimulados com novas expectativas estratégicas e contribuirá com uma boa qualidade de vida no ambiente organizacional.

Palavras-chave: Liderança; características e habilidades do líder; clima e cultura organizacional. 


\section{Abstract}

The present work aimed to describe how leadership, characteristics and skills of the leader, the relationship between leader and its leaders and strategic leadership influence climate and organizational culture. The theoretical basis of the research used leadership discourses focused on the leader as an important element for organizations to obtain a harmonious and healthy environment through climate and organizational culture and for their employees to bring better results to the company.The methodology had an exploratory character with a qualitative point of view, where articles, books and monographs were used, where it is being related in the survey in concepts about what types of characteristics and abilities that leaders can exercise so that the climate and culture of an organization is in perfect condition and that its employees can perform their functions and that the relationships between leaders and leaders are always in favor of the organization's objectives. With this was found through the analyses, that because there is a search for leaders by organizations, they need professionals qualified to improve their results more and more, leading people positively, influencing them with motivation and thus achieve the proposed objectives.It is concluded that, in the business environment it is necessary that the leader obtains the competence to positively influence its members, where the climate and organizational culture are primary factors for the organization, because they will be stimulated with new strategic expectations and will contribute to a good quality of life in the organizational environment.

Keywords: Leadership; characteristics and skills of the leader; climate and organizational culture.

\section{Introdução}

O presente estudo vem possibilitar a ampliação dos conhecimentos sobre liderança, o clima e a cultura organizacional, o entendimento e os seus efeitos gerados na organização. O líder dispõe de um conhecimento fundamental para o benefício da empresa, pois é por meio dele que a mesma poderá acarretar um progresso em todos os setores que constituem, obtendo alto rendimento e motivação entre os membros da organização, sendo fundamental que haja uma avaliação do clima organizacional e possibilitando a satisfação e o bem estar da equipe.

Simonato (2019) em seu site, relata que as empresas e membros de uma organização exigem obter líderes interessados em perceber e entender cada pessoa, líderes que possam ouvir idéias, opiniões e pontos de vistas de sua equipe, 
compartilhe resultados em grupo e que possa, sobretudo, praticar a inteligência emocional.

Todo líder é capaz de ser um administrador, no entanto, nem todo administrador consegue ser um líder. Para exercer a liderança, o indivíduo deve desempenhar uma excelente influência para o comportamento de seus membros, apresentandopara cada um que eles podem ser interdependentes. Esta conexão com os subordinados serve para conquistá-los e consequentemente depositar confiança. Tal motivação é reconhecidapelo nível de interesse que o líder tem em relação as suas necesssidas, identificando seus estímulos, e assim o líder identifica as pessoas que ele pretende liderar (MAXIMIANO, 2011).

A liderança é essencial para o comportamento de uma equipe, com isso o líder poderá direcioná-los a conquistar os objetivos e as metas que a organização precisa (ROBBINS, 2005).

Maxwell e Dornan (2011) adotam a idéia de que a influência é um procedimento para alcançar os objetivos desejados, quando a mesma é utilizada de acordo com a sua habilidade.

O comportamento de um líder é demonstrado por meio de características. Oliveira (2010), demonstra estas características por dois tipos de condutas: a de apoio e a diretiva. Na conduta de apoio, o líder procura ter uma melhor convivência com os seus membros, colocando em destaque o valor humano e emocional da pessoa. A conduta diretiva concede-se na formação de um desenvolvimento operacional e produtivo, focalizando imensamente no trabalho.

Trazendo um foco mais profundo, Oliveira (2010) confirma que a contar do momento que estes comportamentos forem demonstrados serão determinados os estilos de liderança. Cada estilos desses dependerá da maturidade de sua equipe.

De acordo com contexto, o presente estudo com como objetivo descrever as características e habilidades de um líder e como ele pode trazer uma melhoria no ambiente organizacional, e como pode influenciar no clima e na cultura da organização.

Assim, justifica-se a presente pesquisa por entender que a abordagem do conhecimento sobre a liderança e o que os líderes podem fazer numa organização, se encontram num ambiente prejudicado pela falta de comunicação, motivação e 
entendimento entre seus colaboradores, compreendendo que o estudo se faz necessário pois possibilita ampliar os conhecimentos sobre a liderança, clima e cultura organizacional, seus conceitos e efeitos gerados na organização.

\subsection{Liderança Organizacional}

De acordo com Maxwell (2012), a liderança não é uma posição, e sim um processo por meio de dinâmicas usadas em uma equipe que está sempre em desenvolvimento e seu desafio é facilitar o crescimento profissional e criar uma mudança favorável na organização.

Liderar, segundo Hunter (2014, p.115), "é a habilidade de influenciar pessoas para que trabalhem com entusiasmo por objetivos identificados e voltados para o bem comum, com um caráter que inspire confiança e excelência".

Ogbonnia (2007) menciona o tema liderança como a capacidade de agregar e maximizar com sucesso os recursos da empresa, aperfeiçoar os ambientes, para atingir metas sociais ou empresariais. Já Robbins (2005) define liderança como a capacidade de influenciar uma equipe para assim atingir seus objetivos, sendo exercida pelos executivos da organização e por posições com graus de inferioridade.

Sobre o contexto da liderança, Maccoby (2004) e Vergara (2000) corroboram e expressam a importância dos líderes nas empresas, pois sem eles não existiria a liderança.

Cardwell e Tichy (2003) completam que as empresas que objetivarem na construção de novos líderes permanecerão com sucesso no mundo atual e competitivo que as empresas hoje vivem.

Um líder competente deve desenvolver e aperfeiçoar a liderança por meio de características pessoais para assim conseguir excelentes resultados (MAXWELL, 2000).

Assim, Maxwell (2000) menciona ainda que as características que os líderes devem possuir são: caráter, carisma, comprometimento, comunicação, competência, coragem, discernimento, foco, generosidade, iniciativa, ouvir, paixão, atitude positiva, solução de problemas, relacionamentos, responsabilidades, segurança, auto disciplina, ser prestativo, educabilidade e visão. 
Candeloro (2013) descreve que um líder pode manifestar suas características de liderança, mas também afirma que treinando ou desenvolvendo qualidades e competências numa pessoa, certamente se tornará um líder.

Nas concepções de Zenguer e Folkman (2008), os autores relatam que um excelente líder tem seus limites e defeitos, porém não é perfeito, e seus pontos fortes mostram um desenvolvimento de qualidade que faz o relacionar com as pessoas e com os membros da equipe.

Simonato (2019) corrobora destacando as características do líder por meio da letra "l" do alfabeto como ponto chave. O líder precisa "Influenciar" os membros de uma equipe de forma naturalmente, em que o mesmo deve se "Interessar" de forma sincera pelas pessoas e em consequência fica "Interessante" para a equipe, que é onde irão seguir e acatar seus conselhos.

Ainda na concepção de Simonato (2019), o autor constata que além do líder ser um influenciador, é necessário que ele seja um pessoa interessada, por que saberá ouví-los, conhecer as necessidades, os desejos e as mágoas da sua equipe, em que conseguirá influenciá-los de forma objetiva e assim obter ótimos resultados.

Contribuindo com as afirmações apontadas, Nascimento (2008, p.70) relata que "acentuar a liderança é um processo contínuo e interminável. Mesmo assim, nenhum líder jamais chegará ao ponto da perfeição no que diz respeito à perspicácia e à eficácia na liderança", e um bom líder tem que ter qualidades para liderar uma equipe, porém nem sempre está nos cargos mais importantes da empresa, mas tem a incumbência de estar presente em todas as áreas de atuação e em todos os níveis hierárquicos de uma organização (LACOMBE, 2005).

De acordo com as habilidades do lider, Maxwell (2008) relata que todo líder deve possuir uma relação com as mesmas para se tornar um líder potencial.

O líder deve ajustar-se rapidamente às mudanças, compreender as questões mais importantes, enxergar e ter uma visão ampla sobre o ambiente em que está, interagir-se com todas as pessoas da organização, possuir uma boa segurança, ter disposição para servir, encontrar maneiras criativas e inovadoras, colocar a sua equipe sempre em primeiro escalão, possuir um caráter devido à sua competência e ser digno de confiança. 
Zenger e Folkman (2008) relatam que a liderança abrange uma mudança de comportamentos e faz desenvolver a equipe para trazer melhores benefícios para a organização. Com isso, os autores afirmam que os líderes deverão possuir habilidades fundamentais para examinar devidos problemas, tendo uma devida iniciativa para com os liderados, inspirando-os, motivando-os e inovando a cada dia.

Seguindo o mesmo pensamento, Maxwell (2008) completa que a existência do líder é ajudar as pessoas para que elas crescam profissionalmente, com isso motivando-as e inspirando-as irão agregar valores a elas, e Simonato (2019) contextualiza o assunto, quando relata que o líder sabe descobrir cada competência e cada habilidade de seus liderados. O autêntico líder é aquele que sabe exercer uma função de reunir e qualificar cada liderado em seu determinado ambiente de trabalho.

E nessa linha de entendimento, Mintzberg (2010) certifica que para se tornar um líder deve-se aprimorar as habilidades, buscando a fundo conhecimentos e proporcionar oportunidades para que possam se desenvolver e aperfeiçoar suas habilidades de liderança.

\subsubsection{Relaçoes entre Líderes e Liderados}

Em se tratando das relações entre líderes e liderados, Maxwell (2008), relata que os líderes eficazes levam outros consigo para o nível mais elevado de profissionalismo, ou seja, é necessário que a liderança promova a melhoria de cada membro da equipe. Para se obter ótimos resultados, os líderes não devem se manter distante de seus liderados, caso contrário tornar-se-a difícil a realização das tarefas.

A distância impossibilita a aproximação fazendo com que o líder não vivencie as necessidades de seus liderados. Ao compreender esse comportamento e essa liderança distante, o líder desvaloriza cada membro da equipe (MAXWELL, 2008).

Bergamini (2009) corrobora com o contexto acima e afirma que os líderes e seguidores ficam mais propensos a assumir riscos se não houver uma confiança entre as duas partes. Porém, eles são obrigados a atingir metas e resultados e a grande parte dos membros da equipe não considera seu líder como exemplo a ser 
seguido, evitam pedir orientações e não trocam informações pessoais. Então, percebe-se que os líderes preocupam-se mais com resultados do que com o progresso pessoal da equipe, em consequência não haverá uma relação harmoniosa entre líder e liderados, desencadeando assim um relacionamento pessoal.

Canfield e Switzer (2017) evidenciam a importância do líder no relacionamento com sua equipe, seja em qualquer tipo ou nível de atuação, assim o líder saberá entender e se dialogar com seus liderados e em consequência irá influenciá-los. Essa ação é um dos pontos essenciais para se obter uma liderança bem-sucedida.

Contribuindo com o pensamento exposto, Hunter (2006) menciona que não existe uma regra definida de como lidar com os funcionários, mas na função de líderes deve ter uma relação de lealdade, respeito, dignidade e individualidade pois assim, o relacionamento entre as partes se tornará vitoriosa tanto para a empresa quanto para as mesmas e a equipe estará bem estruturada e tudo funcionará melhor e Maxwell (2011) corrobora afirmando que para descobrir como está sendo seu papel de líder você deve perguntar para sua equipe como está a sua liderança, verificar se os liderados estão mudando seu perfil, se as pessoas que estão ao seu redor estão no caminho do sucesso e se desenvolvendo. Tendo todas essas perguntas respondidas o gestor saberá como está seu nível de liderança.

Nessa linha de liderança, há que se entender sobre a liderança estratérgica, que segundo Gerras (2010), a liderança estratégica é um modelo em que o líder influencia, motiva e inspira para a execução a longo prazo de um futuro desejável, adequando a cultura organizacional, adquirindo recursos, planejamento e organização de mudanças por meio de políticas e diretrizes. O modelo de liderança é enérgico por natureza e exige uma experiência elevada de empenho e envolvimento de cada membro da equipe.

\subsection{Liderança Estratégica}

Para Rowe (2002) o conceito de liderança estratégica é influenciar pessoas de uma forma voluntária fazendo-as tomarem decisões que melhorem a viabilidade 
de médio e longo prazo da organização e ao mesmo tempo mantendo a estabilidade financeira da empresa em curto prazo de tempo.

Conforme Teixeira (2011) para haver o sucesso da organização, é necessário a implementação de uma estratégia bem desenvolvida e planejada, para que os gestores possam atuar em diversos níveis, e ter a capacidade de influenciar e encorajar os seus liderados a atingirem seus objetivos.

Chiavenato e Sapiro (2010) corroboram contextualizando que a liderança estratégica requer que a organização administre as pessoas de acordo com os próprios recursos que ela tem, e que esses líderes devem praticar a influência no comportamento das pessoas num ambiente desconhecido e imprevisível e Blanchard (2010) conclui que a liderança é o que irá impulsionar a organização a um elevado desempenho, e destaca o diálogo entre a liderança estratégica e a liderança operacional, para causar uma impressão de paixão em cada integrante da equipe, assim acarretará um afeto no cliente e na força vital da empresa.

Quando se trata do ambiente organizacional, considera-se como pressuposto a cultura organizacional.

\subsection{Cultura Organizacional}

Chiavenato (2012) certifica que a cultura organizacional é uma característica tradicional ou de costume que tem como objetivo a resolução dos problemas de adaptação externa e interna da empresa, que pode ser classificado como válido e desejável que deve ser comunicado aos novos colaboradores possam perceber, pensar e sentir como os problemas podem ser resolvidos.

Complementando ainda com seu entendimento, Chiavenato (1999) relata que a cultura organizacional é produzida no decorrer do tempo e passa a influenciar de forma decisiva nas práticas do dia a dia, estabelecendo um conjunto de representações mentais num sistema harmonioso de significados que incorpora todos os integrantes de uma organização em torno dos mesmos propósitos e em todas as formas de como se proceder. 
A cultura organizacional é uma conexão entre a atualidade e o que aconteceu em decorrido acontecimento e sempre contribuirá para a conservação e a união da organização.

Conforme Maximiano (2010), a cultura organizacional inclui diversos fundamentos, que foram desenvolvidos por profissionais do passado e propagados pelos atuais profissionais. Aqueles novos colaboradores que comporem a organização, devem ter conhecimento da cultura da empresa e aprender a se comportar de acordo com os elementos culturais.

Shein (2009) corrobora afirmando que a cultura é a união de estruturas, rotinas, regras e normas que orientam e resumem o comportamento da organização, sendo um evento habitual e frequentemente praticado e elaborado por interações entre os membros da empresa e moldado por comportamento de liderança.

Assim, Shein (2009) conclui que a cultura organizacional e a liderança estão entrelaçadas, pois se a permanência da equipe estiver comprometida por causa de uma cultura desordenada, é dever e função dos líderes em todos os níveis da organização admitir e fazer algo em relação a essa circunstância, então pode-se inferir que a cultura organizacional é um procedimento de aprendizagem de um grupo que é relativamente inspirado e motivado pelo comportamento do líder.

\subsection{Clima Organizacional}

O Clima Organizacional, de acordo com Chiavenato (2012) retrata que o clima organizacional refere-se aos fatores internos da instituição, que levam ao estímulo de diferentes motivos de motivação nos seus colaboradores. Essa ferramenta é produtiva quando possibilita satisfazer as necessidades pessoais dos membros da empresa, fornecendo-os melhoramento da moral interna, e tornando prejudicial quando proporciona a frustração.

Segundo Maximiano (2000) o clima organizacional é constituído por emoções de vivências que as pessoas compartilham no ambiente empresarial e isso pode afetar positivamente ou negativamente na motivação e satisfação no exercício de suas funções. Sentimentos negativos provocam diminuição no desempenho das atividades dos colaboradores, gerando um clima de tensão, medo, tristeza e 
ansiedade. Sentimentos positivos provoca impacto positivo, criando em seu ambiente alegria, motivação e amizades verdadeiras.

Conforme Chiavenato (2002), o autor afirma que uma motivação baixa por causa de frustração ou obstáculos à satisfação dos desejos e necessidades das pessoas envolvidas na empresa, o clima organizacional pode cair, caracterizando vários distúrbios como: depressão, desinteresse, apatia, insatisfação, etc., sendo na maioria dos casos chegar a estados de agressão, confusão, inconformidade, etc. onde as equipes se defrontam abertamente com a organização.

Por fim, corroborando com Chiavenato (2002), Maximiano (2000) menciona que o clima é construído por comportamentos de afinidade que os colaboradores compartilham entre si à respeito da organização e que aparentam positivamente ou negativamente devido a sua satisfação e motivação para o exercício da profissão.

\section{Metodologia}

$\mathrm{Na}$ elaboração do presente artigo, foi relacionado levantamentos de acordo com o tema, seus objetivos propostos e sendo abordado com o ponto de vista e o pensamento de autores e pesquisadores por meio de uma pesquisa bibliográfica.

Refere-se em uma pesquisa qualitativa e que está sendo relacionada no levantamento em conceitos sobre as quais od tipos de características e habilidades que os líderes podem exercer para que o clima e a cultura de uma organização esteje em perfeitas condições, e para que seus colaboradores possam desempenhar suas funções e que o relacionamento entre ambos (líderes e liderados), estejam sempre a favor dos objetivos da empresa.

Os autores mencionados como referências no assunto sobre liderança foram John Calvin Maxwell ${ }^{1}$ e Idalberto Chiavenato ${ }^{2}$ nos conteúdos de clima e cultura organizacional, em que as mesmas foram compostas de análises de referências teóricas por meio de livros, artigos, monografias, etc.

\footnotetext{
1John Calvin Maxwell -autor cristão evangélico, conferencista e pastor que escreveu mais de 60 livros, centrado principalmente em liderança. Bacharel na Ohio Christian University, em 1969. Concluiu o mestrado em Azusa Pacific University, em 1989 e o doutorado no Ministério no Fuller Theological Seminary, 1993 (Seminário Teológico Fuller, 1993) e também recebeu cinco doutoramentos honorários de divindade (incluindo aquelas da Califórnia Graduate School de Teologia E Liberty University).

${ }^{2}$ Idalbeto Chiavenato - autor nacionalmente conhecido e respeitado na área de Administração, Doutor e Mestre em Administração, especialização em Psicologia Educacional.
} 
A pesquisa qualitativa segundo Creswel (2007) nos diz que por ser uma fonte direta de dados, em que o pesquisador é o elemento fundamental na elaboração dos assuntos descritivos. Com isso, Creswel (2007) destaca que a atenção com o processo é mais importante que o produto em si, isto é, o interesse do pesquisador ao averiguar um determinado erro, é investigar como ele se define nas suas atividades e nas interações do dia a dia.

Godoy (2005) evidencia alguns pontos essenciais para que uma pesquisa qualitativa contenha uma ótima qualidade, tais como: confiabilidade, mostrar resultados confiáveis; viabilidade, apresentar uma explicação de forma que o leitor tenha um entendimento do assunto em outro com textura de idéias; credibilidade, no método de desenvolvimento que a pessoa irá utilizar na pesquisa; fidedignidade dos resultados coletados na pesquisa; explanação cuidadosa dos método da pesquisa, detalhando o que foi utilizado no estudo do tema e, finalmente, a relevância nas questões de pesquisa, com relação aos estudos anteriores.

$\mathrm{Na}$ visão de Denzin e Lincoln (2006), a expressão qualitativa traz um destaque a respeitosobre as qualidades das organizações e com relação sobre os métodos que não podem ser explicados ou medidos na prática em termos de volume, quantidade, intensidade ou frequência, consequentemente o conhecimento quantitativo enfatiza $o$ ato de medir e examinar as relações causais entre circunstâncias, e não os procedimentos.

\section{Resultados e Discussão}

As análises foram realizadas por meio da interpretação por meio da subjetividade, que produziram elementos suficientes às conclusões pelo método teórico levantado.

Compreender as fronteiras entre a transformação e o desenvolvimento da subjetividade demanda uma percepção sensível dos pesquisadores, que devem estar protegido por uma exatidão teórica que lhe permitam determinar inteligibilidade em meio à complexidade humana e seus procedimentos evolutivos nas organizações.

Assim, compreendeu-se com base ao tema apresentado, que Carnegie (2012, p.25) afirma que "o líder deve aprender a ser um mentor, deve concentrar em ajudar 
as pessoas a dar o que têm de melhor e a desenvolver os próprios talentos, encorajando as pessoas a usarem a própria iniciativa e discernimento".

Com isso Maxwell (2012, p.21) corrobora que:

Líderes tornam-se grandes, não por causa do poder, mas por causa da habilidade em capacitar as pessoas. Usam a posição, relações e produtividade para investir em seus seguidores e desenvolvê-los até que esses seguidores se tornem líderes por conta própria.

Como explicitado nos estudos, o conceito de liderança é conduzir pessoas de forma positiva e com motivação para alcançarem os objetivos da equipe e da organização, mas não podemos esquecer que, o estudo sobre liderança não serve só para identificar os estilos de liderança de cada líder, mas identificar as táticas de influência mais eficazes para conduzir as equipes a conquistar os melhores resultados.

Essa informação confirma com os resultados obtidos nos estudos de Kernberg (2000), que afirma que o papel do líder é estimular os liderados nas funções de convívio com o grupo para serem aceitas e reconhecidas e nas quais, são baseadas em seus procedimentos e suas percepções. A personalidade do líder abrange suas características de carisma e suas características autoritárias.

Dando continuidade no assunto de líder e liderados, pode-se também perceber como o relacionamento entre líder coach e coachee, em que coaching ${ }^{3}$ é um assunto de liderança bem abordado nos últimos tempos, em que o profissional coach instrui o liderado para que possa aprender e desenvolver a criatividade e a iniciativa, em que o líder possa mobilizar o grupo, equipe ou a pessoa (liderado) por meio do aprendizado contínuo, construindo assim a autoconfiança, tratando cada liderado de forma única e mostrando a cada um que todos são iguais.

Para confirmar o assunto acima, Matta (2012, p. 15) define o coaching como:

Um processo que visa elevar a performance de um indivíduo, do grupo ou da empresa, aumentando os resultados positivos por meio de metodologias, ferramentas e técnicas cientificamente validadas, aplicadas por um profissional habilitado (o coach), em parceria com o cliente (o coachee).

Nos dias atuais, o relacionamento entre líder e liderados tem sido um divisor de águas no ambiente organizacional. Colaboradores deixam suas empresas devido um mal procedimento ou uma má atitude de seus líderes, em que o funcionário não

\footnotetext{
${ }^{3}$ Para o português significa treinamento, então, o coach é um treinador de equipes onde desenvolve a pessoa ou o profissional a definir suas metas e alcançar objetivos.
} 
tem sido valorizado e respeitado em seu ambiente de trabalho, com esse resultado há um aumento do índice de rotatividade, diminuição do desempenho e da produtividade dos colaboradores dentro da empresa em que o clima organizacional fica propenso a desmotivação.

Assim, os estudos de Robbins (2002) afirma que a performance e a satisfação do funcionário tendem a ser sem dúvida influenciados pelas atitudes e comportamentos do líder, consequentemente a própria estrutura organizacional da empresa pode desmotivar um colaborador, como também o tipo de liderança exercido pelos seus líderes.

Corroborando com Robbins (2002), Carnegie (1981) contextualiza que as pessoas têm desejos de se sentir valorizados, e não é um simples desejo, elas precisam suprir suas necessidades e quando não conseguem saciar seus anseios, ficam decepcionados, desmotivados e não conseguem ter a vontade de executar suas funções na empresa.

Compete destacar que os modelos de liderança são estilos próprios de cada líder, seja ele autocrático, democráctico ou situacional (aquele que se molda de acordo com o que cada colaborador tem como ponto forte). Cabe ressaltar que a tática de pressão não contribui positivamente para a equipe e a organização, pois o resultado interfere na motivação, autoconfiança e na disposição do trabalho em equipe. Como foi demonstrado durante a pesquisa, algumas habilidades que o líder deve possuir para conduzir um grupo de pessoas, pois só com competências é que pode-se motivar e inspirar pessoas para conquistar positivamente os objetivos.

Para colaborar com o que foi relatado anteriormente, Chiavenato (2005) menciona que não depende só do colaborador para se conseguir talento, as organizações devem motivar, inspirar, desenvolver e recompensar para que elas alcancem os objetivos propostos.

Entendeu-se que o líder estratégico tem o poder de trabalhar com todas as táticas e diferenças de cada membro e faz delas o seu sucesso. O autor sabe reconhecer o valor de cada pessoa para o exercício da profissão e também a importância do profissional para a organização. O líder estratégico estará sempre disposto a trazer ótimos resultados, mostrando ser eficiente e eficaz a cada missão. 
Esse relato vai de encontro com Carnegie (2014, p.9), que confirma que "o ser humano passou a ser valorizado como o ativo mais importante das empresas e, saber impulsionar e direcionar esses talentos passou a ser prioridade".

Nessa linha, muitas empresas tentam a cada dia obter profissionais capacitados para desempenhar suas funções de modo que agregue benefícios para a mesma e também para seus colaboradores. Hoje o cargo de gestor tem o dever tem como dever gerir pessoas e seus processos onde busca a cada dia as melhorias para a organização, propondo inovações e administrando da melhor forma possível os recursos da empresa. Já o líder, tem como seu perfil de inspirar, motivar e influenciar as pessoas, fazendo de uma forma que elas executem o seu serviço de forma natural, sem nenhuma obrigação.

Mediante a afirmação acima, Hatakeyama (1995, p. 3) diz que "o gerente é mais do que [...] a pessoa responsável pela execução da instrução que vem de cima. O seu papel é mais dinâmico [...].

Por fim, completando com o entendimento, Maxwell (2012) defendeu que a atitude do líder vem a partir do desenvolvimento da influência, que pode ser realizado por qualquer profissional independentemente do cargo que exerce, pois esse profissional estará preparado para desenvolver qualquer função ligado a liderança, com isso irá encarar os desafios apresentados a partir do seu comportamento, e não somente por pessoas que não tem um cargo de chefe.

\section{Conclusão}

O propósito da pesquisa foi apresentar por meios de estudos a importância da liderança dentro de uma organização no qual o clima e cultura organizacional são fatores primordiais para a organização, e como elas podem influenciar no desempenho de cada colaborador da empresa.

Percebeu-se que no ambiente empresarial é necessário que o líder obtenha a competência de influenciar de maneira positiva de seus membros e conduzí-los a alcançar resultados propostos e que serão relevantes para a empresa. A função do líder é aperfeiçoar os integrantes da equipe, por meio da capacidade de estabelecer prioridades e apontar uma orientação correta, demonstrando suas qualidades e 
habilidades, dentre eles o entusiasmo, a flexibilidade, a persistência, a autoconfiança, a iniciativa e o otimismo.

O gestor tem seu papel na organização, pois fortalece na busca de estratégias efetivas que ajudem a desenvolver e impulsionar constantemente 0 sucesso dos negócio da empresa. O profissional quando domina todos os conhecimentos técnicos, é exemplar em sua área de atuação, é promovido gestor do seu departamento e por questões semelhantes, acontece que muitos não tem a habilidade de gerir pessoas, sua inexperiência para liderar acaba minando resultados e não consegue projetar bons resultados para organização.

Nas organizações tem-se colaboradores de vários perfis, uns com perfil de gestores e outros com perfil de líderes, podemos citar que pessoas com perfil líderes podem exercer funções mesmo não estando em algum tipo de posição de liderança na hierarquia da empresa, pois exercem a influência em seus colegas não pelo que dizem mas pelas atitudes em exercício da profissão. O líder compartilha idéias e estimula as pessoas por meio de crenças comuns que ambos possuem, consequentemente serão admirados por não existir uma afeição de obrigação em seu ambiente de trabalho.

Compete ao líder adaptar-se às novas realidades e ser capaz de comandar todos os seus membros pelos procedimentos de mudanças que progressivamente são fundamentais para as organizações em que os mesmos se encontrem dispostos para modificar as novas ameaças em oportunidades.

Observou-se que com relação à cultura organizacional, cada organização tem sua cultura própria, isso se deve o caminho percorrido com seus obstáculos, fracassos e vitórias confrontados dia após dia na empresa.

A liderança é outro elemento que tem influência no clima, tendo o líder em seu papel a harmonia e o relacionamento grupal da equipe, com devido reconhecimento e valorização dos integrantes da empresa, mantendo objetivos importantes, com a determinação de uma causa comum.

Quando fala-se em liderança também falamos em líder coach pois são duas expressões que andam juntas e tem o mesmo propósito, instruir, motivar, elevar, incentivar e lidar com equipes e pessoas. 
Concluiu-se que para diminuir a rotatividade e aumentar o desempenho e a produtividade de colaboradores nas organizações os líderes tem o dever de promover a motivação, a elevação e incentivo da equipe de trabalho, criando um clima de bem estar e colaboração gerando a qualidade de vida no ambiente organizacional bastante positivo e saudável, pois assim difundirá uma visão de futuro em que estimulará seus integrantes com novas expectativas estratégicas para a melhoria da própria organização e contribuirá de forma importante para a academia e para a prática mercadológica.

Por fim, com o intuito de dar continuidade na busca de melhoria nas organizações, recomenda-se que a presente pesquisa seja dado prosseguimento com futuras investigações, para a melhoria do conhecomento e assim corroborar com novos resultados revelados por esta.

\section{Referências}

BLANCHARD, Ken. Um Nível Superior de Liderança. 4ª Ed. Lisboa: Editora Actual,2010.

BERGAMINI, Cecília Whitaker. Liderança: Administração do sentido. 2. ed. São Paulo: Atlas, 2009.

CANDELORO, Raúl. As 8 características de um líder de vendas de sucesso. Edição 229. Curitiba: 2013.

CANDIELF, Jack e SWITZER, Janet. Os princípios do sucesso. 8. ed. Barcarena. Editora Marcador, 2017.

CARDWELL, N.; TICHY, N. M. Feitas para o sucesso: como grandes líderes ensinam suas empresas a vencer. Rio de Janeiro: Campus, 2003.

CARNEGIE, Dale. Como Fazer Amigos e Influenciar Pessoas: Tradução de Fernando Tudo de Souza. 45. Ed. 1981.

. Como Fazer Amigos e Influenciar Pessoas: Tradução de Fernando Tude de Souza. 45. Ed. 1981. Disponível em: <http://www.teresacoutinho.com/ebooks/comofazeramigos.pdf>. Acesso em 12 de Jan de 2012.

Liderança: como superar-se e desafiar outros a fazer o mesmo. 2. ed. São Paulo: Companhia Editora Nacional, 2014.

CHIAVENATO, Idalberto. Gestão de Pessoas: O novo papel dos recursoshumanos nas organizações. 9ª Tiragem. Rio de Janeiro: Campus, 1999.

. Recursos Humanos. Ed. Compacta. $7^{\mathrm{a}}$ ed. São Paulo: Atlas, 2002. 
Comportamento organizacional: A dinâmica do sucesso das organizações. São Paulo: Pioneira Thomson Learning, 2004.

Comportamento Organizacional: A dinâmica do sucesso das organizações. 2. ed. Rio de Janeiro: Elsevier, 2005.

. Recursos humanos: o capital humano das organizações, ed. 9, Rio de Janeiro: Elsevier editora, 2009.

. Gerenciando com pessoas: transformando o executivo em um excelente gestor de pessoas. 9. reimp. Rio de Janeiro: Elsevier, 2012.

CHIAVANETO, I.; SAPIRO, A. Planejamento Estratégico. São Paulo: Elsevier, 2010.

CRESWEL, J. W. Projeto de pesquisa: método qualitativo, quantitativo e misto. 2. ed. Porto Alegre: Artmed, 2007.

DENZIN, N. K. e LINCOLN, Y. S. Introdução: a disciplina e a prática da pesquisa qualitativa. In: DENZIN, N. K. e LINCOLN, Y. S. (Orgs.). O planejamento da pesquisa qualitativa: teorias e abordagens. 2. ed. Porto Alegre: Artmed, 2006. p. 1541.

GUERRAS, Stephen J.Iniciador Estratégico para Liderança. Departamento de comando, liderança e gestão do colégio de guerra do exército dos Estados Unidos. $3^{\text {a }} \mathrm{Ed}, 2010$.

\section{GODOY, A. S. Refletindo sobre critérios de qualidade da pesquisa}

qualitativa. Revista Eletrônica de Gestão Organizacional, v. 3, n. 2, p. 81-89, mai./ago. 2005.

HATAKEYAMA, Y. A revolução dos gerentes. Belo Horizonte: Fundação Christiano Ottoni, 1995.

HUNTER, James C. Como se tornar um líder servidor. Rio de Janeiro: Sextante, 2006.

De volta ao mosteiro: 0 monge e o executivo falam de liderança e trabalho em equipe. 1. ed. Rio de Janeiro: Sextante. 2014.

KERNBERG, Otto F. Ideologia, conflito e liderança em grupos e organizações. Porto Alegre: Artes Médicas Sul, 2000.

LACOMBE, Francisco Jose Masset. Recursos humanos: Princípios e tendências. São Paulo: Saraiva, 2005.

MACCOBY, M. Por que seguimos os líderes: o poder da transferência. Harvard Business Review, v. 82, n. 9, p. 59-68, set. 2004.

MATTA, Villela da. Personal e Professional Coaching: Livro de metodologia. Rio de Janeiro: SBCoaching, 2012.

MAXIMIANO, Antonio Cesar Amaru. Teoria Geral da Administração: da escola científica à competitividade na economia globalizada. $2^{\underline{a}}$ ed. São Paulo: Atlas, 2000. 
Teoria geral da administração: da revolução urbana à revolução digital. 6 . ed. 7. reimp. São Paulo: Atlas, 2010.

. Fundamentos de Administração: manual compacto para as disciplinas TGA e introdução à administração. 2. ed. São Paulo: Atlas, 2011.

MAXWELL, J. C.; DORNAN, J. Liderar é influenciar. Rio de Janeiro: Thomas Nelson Brasil, 2011.

MAXWELL, John C. As 21 indispensáveis qualidades de um líder / John C. Maxwell; tradução de Josué Ribeiro. São Paulo: Mundo Cristão, 2000.

. O livro de ouro da liderança. Rio de Janeiro: Thomas Nelson Brasil, 2008.

Os 5 níveis da liderança. Traduzido por Luís Aron de Macedo. 1. edição. Rio de Janeiro: CPAD, 2012.

Líder 360: Como desenvolver seu poder de influência a partir de qualquer ponto da estrutura corporativa. 2. ed. Rio de Janeiro: Thomas Nelson Brasil, 2012.

MINTZBERG, Henry. Managing: desvendando o dia a dia da gestão. Porto Alegre. Bookman, 2010.

NASCIMENTO, E. Comportamento Organizacional. Curitiba: IESDE Brasil S.A., 2008.

OLIVEIRA, Marcos A. Comportamento organizacional para a gestão de pessoas: como agem as empresas e seus gestores. São Paulo: Saraiva, 2010.

OGBONNIA, K.S. Sistema de partidos políticos e liderança eficaz na Nigéria: Abordagem de contingência. Dissertação. Walden University, 2007.

ROBBINS, Stephen P. Comportamento organizacional. Tradução: Reynaldo Marcodes. 9. ed. São Paulo: Prentice Hall, 2002.

Comportamento Organizacional. Tradução Reynaldo Marcondes. 11. ed. São Paulo: Pearson Prentice Hall, 2005.

ROWE, W. Glenn. Liderança estratégica e criação de valor [Em Linha]. Fórum AME de Estratégia e liderança, 2002.

SHEIN, Edgar H. Cultura organizacional e liderança. Tradução Ailton Bomfim Brandão; revisão técnica Humberto Mariotti. São Paulo: Atlas, 2009.

SIMONATO, Marcelo. Liderança é como um namoro: o líder que se interessa pela equipe se torna interessante para ela também. Prod. São Paulo, 2019. Disponível em: http://marcelosimonato.com/lideranca-e-como-um-namoro-o-lider-que-seinteressa-pela-equipe-se-torna-interessante-para-ela/Data de acesso: 01/12/2019.

SALOMAO, P. E. A. et al. As Tecnologias de Informação e Comunicação (TIC) no ensino superior. Revista Multidisciplinar do Nordeste Mineiro, v. 1, 2018.

TEIXEIRA, Elson Adalberto. Teoria geral da administração e prática: TGA \& P. 1. ed. Rio de Janeiro: Editora FGV, 2002. 
TEIXEIRA, Sebastião. Gestão estratégica. Lisboa: Escolar, 2011.

VERGARA, S. C. Gestão de pessoas. São Paulo: Atlas, p. 73-105, 2000.

ZENGER, John H.; FOLKMAN, Joseph. Desenvolva sua capacidade de liderança: 24 estratégias para melhorar suas habilidades como líder. Rio de Janeiro: Sextante, 2008. 